\title{
On Vegetation Clustering, Localized Bare Soil Spots and Fairy Circles
}

\author{
M. Tlidi, R. Lefever, and A. Vladimirov
}

\begin{abstract}
We present a model and nonlinear analysis which account for the clustering behaviors of arid vegetation ecosystems, the formation of localized bare soil spots (sometimes also called fairy circles) in these systems and the attractive or repulsive interactions governing their spatio-temporal evolution. Numerical solutions of the model closely agree with analytical predictions.
\end{abstract}

\section{Introduction}

It is common in semi-arid or arid regions to encounter landscapes where the vegetation cover is non-uniform and exhibits large-scale structures, generically called "vegetation patterns" $[1,2]$. The terms "semi-arid" and "arid" refer here to climatic conditions where water resources are scarce. More precisely, they mean that the potential evapo-transpiration largely exceeds the water supply provided by rainfall. At the individual plant level, this hydric stress affects plants' growth and survival; at the community level, it generates clustering effects that cause the formation of vegetation patches. The outcome is a spatial "differentiation" of the landscape into a "mosaic" of poorly vegetated and strongly vegetated domains, accompanied by a redistribution of the ecosystem water resources benefiting the vegetated domains.

\footnotetext{
M. Tlidi

Université Libre de Bruxelles, Optique Nonlinéare Théorique, CP 231, Campus de la Plaine, B-1050 Bruxelles, Belgium, mtlidi@ulb.ac.be

R. Lefever

Université Libre de Bruxelles, Service de Chimie Physique et Biologie Théorique, CP 231, Campus de la Plaine, B-1050 Bruxelles, Belgium, rlefever@ulb.ac.be

A. Vladimirov

Weierstrass Institute for Applied Analysis and Stochastics, Mohrenstrasse 39, D-10117 Berlin, Germany, vladimir@wias-berlin.de
}

Tlidi, M. et al.: On Vegetation Clustering, Localized Bare Soil Spots and Fairy Circles. Lect. Notes Phys. 751, 381-402 (2008) 
It is now generally admitted that this adaptation to hydric stress involves a symmetry-breaking modulational instability, whose causes are intrinsic rather than extrinsic, to the vegetation, i.e., they result from the vegetation dynamics itself, rather than from the imposition on this dynamics of some external (environmental), pre-existing spatial periodicity or anisotropy $[3,4,5,6,7,8,9,10,11,12]$. Well-known patterns of this type are the banded, periodic vegetation covers, often labeled with the picturesque name of tiger bush, which can be observed in many arid regions on the earth. The intrinsic, self-organizing mechanism mentioned above provides a unified explanation for the diverse phenotypical characteristics displayed by vegetation patterns. In particular, it predicts the properties of periodic patterns and the sequence of symmetry-breaking transitions, which such patterns undergo as a function of aridity. For example, it accounts for the observation that the size (wavelength) of the heterogeneities increases with the degree of aridity, while, simultaneously, the symmetry of the patterns transforms from $\pi$-hexagonal into stripes and, finally, into 0-hexagonal $[3,6,13]$. Remarkably, besides the behavior of periodic patterns, the same mechanism predicts the possible existence of aperiodic, localized structures (sometimes called dissipative solitons). They consist either of localized patches of vegetation, randomly distributed on bare soil [14, 15] or, on the contrary, of localized spots of bare soil (LBS), randomly distributed in an otherwise uniform vegetation cover.

In this chapter, we shall focus our attention on LBS-type patterns. Welldocumented examples are the so-called woodland glades, a label referring to isolated, open spaces of herbaceous vegetation (sometimes even of bare ground) surrounded by woody vegetation. Woodland glades are permanent structures, and they can be observed worldwide, even in non-arid climates. They may support particular plants, and they are zones of attraction for various animal species which are adapted to woodland and which take advantage of these sunny and dry open areas. Understanding their formation and maintenance is an important ecological issue. Other fascinating, puzzling and well-documented examples of LBS patterns are the fairy circles or fairy rings discovered in the Namib desert (see Fig. 1). These circular areas, devoid of any vegetation, can reach diameters of up to $14 \mathrm{~m}$. This exceeds the size of the tall grasses surrounding them by more than one order of magnitude. In a recent study, van Rooyen et al. [16] have made an in-depth investigation of the strengths and shortcomings of several hypotheses concerning their origin. These authors have been able to rule out external causes, such as the possible existence of localized radioactive areas unsuited for the development of plants, or a link between fairy circles and the activity of termites. Among the other hypotheses they considered (notably, the release of allelopathic compounds and the possible existence of stimulatory or inhibitory influences due to interactions between different plant species), none was found which satisfactorily fitted their experimental observations. Our objective, in regard to this context, is to investigate the generic properties and evolving behaviors which can be predicted for LBS patterns on the basis of the conceptual framework and treatment developed previously for the study of periodic vegetation patterns. 


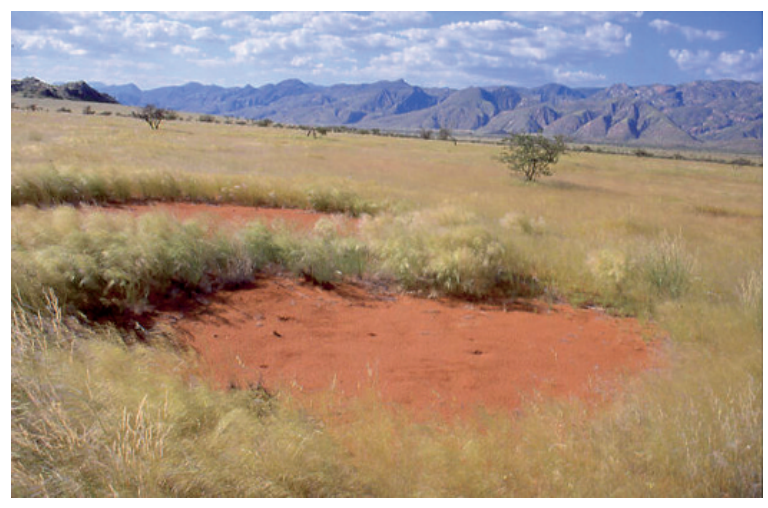

Fig. 1 Example of the fairy circles occurring in the pro-Namib zone of the west coast of southern Africa [16] (photography: courtesy of M. Johnny Vergeer)

The starting point of our approach is a modified version of the integro-differential propagator-inhibitor model (PI model) originally introduced to explain the formation of tiger bushes [3]. The basic hypotheses of the PI model are summarized in Sect. 2. The modifications introduced in its present version have the objectives (i) to formulate more precisely the relationship between the plants' aerialsubterranean structures and the facilitative or competitive feedbacks which influence their dynamics at the community level; and (ii) to incorporate into the model an explicit formulation of the plants' spatial propagation (e.g., by seed dispersion), so that the interrelations between the "transport" phenomena taking place in the ecosystem and the growth/decay dynamics of its constitutive vegetation are accounted for in a better manner. In Sect. 3, we derive, from the original integrodifferential equation describing the dynamics in general, a nonlinear fourth-order partial differential equation which constitutes a convenient, appropriate approximation in the weak-gradient limit, where the size of spatial heterogeneities at the community level greatly exceeds the size of individual plants. The conditions under which vegetation clustering and localized bare spots appear are discussed in Sects. 4 and 5. The homoclinic solutions modeling LBS patterns are then evaluated analytically. To begin with, the case of localized, isolated single spots is treated; the solutions representative of this situation are constructed, and their properties are discussed and compared with the results of numerical solutions. In Sect. 6, the situation where bare spots are close enough to interact is studied. This question, which is of considerable interest, has been investigated in other contexts $[17,18,19,20,21,22,23,24]$. We demonstrate here that such interactions induce the appearance of a "force" between LBS, which may be attractive or repulsive, depending on the distance separating the interacting spots. These results are established analytically and confirmed by numerical simulations. 


\section{Mean-Field Model of Vegetation Evolution}

Let us consider a plant community established on a spatially uniform territory, and suppose that a dominant species accounts for most of the community bio-mass. Let $L_{\mathrm{a}}$ be the radial extension of a mature individual belonging to this species, $S=\pi L_{\mathrm{a}}^{2}$ be a territorial surface element centered on a given spatial point, $\mathbf{r}$, and $m_{\max }$ be the maximal amount of bio-mass that the community could produce on the surface element $S$ in the absence of vegetation decay. We imagine that we redistribute the bio-mass $m_{\mathrm{p}}(\mathbf{r}, t)$ contained in $S$ uniformly over it, then we define the normalized bio-mass at the point $\mathbf{r}$ for time $t$ as $b(\mathbf{r}, t)=m_{\mathrm{p}}(\mathbf{r}, t) / m_{\max }$ and model its spatiotemporal evolution by the logistic equation

$$
\begin{aligned}
\partial_{t} b(\mathbf{r}, t)= & k_{1} b(\mathbf{r}, t)-k_{2} b(\mathbf{r}, t) \\
& +D \int\left[\Phi_{\text {in }}\left(\left|\mathbf{r}^{\prime}\right|\right) b\left(\mathbf{r}+\mathbf{r}^{\prime}, t\right)-\Phi_{\text {out }}\left(\left|\mathbf{r}^{\prime}\right|\right) b(\mathbf{r}, t)\right] \mathrm{d} \mathbf{r}^{\prime},
\end{aligned}
$$

where the unit of time has been set equal to the characteristic time of the growth process. The first two terms in (1) account for the bio-mass gains and losses that the surface element $S$ undergoes due to the natural growth and decay of the plants. The third term accounts for the vegetation spatial propagation via seed dispersion and/or other natural mechanisms. The integration extends over the whole of the territory, which we suppose to be infinitely extended, and $\Phi_{\text {in }}$ and $\Phi_{\text {out }}$ are the dispersion kernels weighting the incoming and outgoing seed fluxes between neighboring points, according to their separation, $\left|\mathbf{r}^{\prime}\right|$. Further, $D$ is a phenomenological constant which fixes the rate of propagation of the vegetation, while $k_{1}$ and $k_{2}$ are kinetic coefficients which take into account the plant-to-plant feedback effects resulting from the communal organization of the vegetation. As such, we suppose that they are mean-field state functions which can be written as:

$$
k_{1}=[1-b(\mathbf{r}, t)] \mathscr{M}_{\mathrm{f}}(b(\mathbf{r}, t), t), \quad k_{2}=\mu \mathscr{M}_{\mathrm{c}}(\mathbf{r}, t) .
$$

The phenomenological constant, $\mu$, is the decay to growth rate ratio in the absence of interactions, i.e., under conditions where the vegetation spatio-temporal dynamics is reduced to that obeyed by isolated plants, while $\mathscr{M}_{\mathrm{f}}(\mathbf{r}, t)$ and $\mathscr{M}_{\mathrm{c}}(\mathbf{r}, t)$ are mean-field factors describing plant-to-plant interactions. By definition, these factors are equal to 1 in the case of isolated (or non-interacting) plants, and greater than one when interactions cannot be neglected. In other words, they enhance the rate of the processes which they influence, and therefore we call them the mean-field enhancing factors. $\mathscr{M}_{\mathrm{f}}(\mathbf{r}, t)$ describes interactions facilitating growth. ${ }^{1}$ They are associated with the plants' aerial structures and involve, notably, a reciprocal sheltering of neighboring plants against climatic harshness, as well as a communally more favorable management of vital resources. They extend over distances of the order

${ }^{1}$ As well as seed production and germination [26]. 
of $L_{\mathrm{a}}$ (e.g., the crown radius of mature trees). On the other hand, $\mathscr{M}_{\mathrm{c}}(\mathbf{r}, \mathbf{t})$ is associated with competitive plant-to-plant interactions enhancing vegetation decay. They predominantly involve the plants' roots system, or rhizosphere. Accordingly, they operate over distances of the order of the rhizosphere radius, $L_{\mathrm{r}}$. Their competitive nature results from the fact that the rhizosphere activity of a plant tends to deprive its neighbors of vital resources, e.g., water [25]. The logistic factor, $[1-b(\mathbf{r}, t)]$, of the kinetic constant $k_{1}$ accounts for the existence of an upper limit, $m_{\max }$, which the bio-mass of a surface element, $S$, cannot exceed. The latter is specific to the vegetation.

The following considerations then determine the choice of the mathematical expressions of the rate-enhancing factors:

(i) By definition, $\mathscr{M}_{\mathrm{f}}(\mathbf{r}, t)$ and $\mathscr{M}_{\mathrm{c}}(\mathbf{r}, t)$ are increasing, functions of the vegetation density. They take values in the interval $[1, \infty$, and they are equal to 1 in the zero-density limit,

$$
\lim _{\rho\left(\mathbf{r}+\mathbf{r}^{\prime}, t\right) \rightarrow 0} \mathscr{M}_{l}(\mathbf{r}, t)=1, \quad \imath=f, c,
$$

where the dynamics of isolated plants must be recovered.

(ii) To fulfill condition (i), we postulate that the rate-enhancing factors depend exponentially upon the vegetation density, with the following form:

$$
\mathscr{M}_{l}(\mathbf{r}, t)=\exp \left(\chi_{l} \int \Phi_{l}\left(\left|\mathbf{r}^{\prime}\right|, L_{l}\right) b\left(\mathbf{r}+\mathbf{r}^{\prime}, t\right) \mathrm{d} \mathbf{r}^{\prime}\right), \quad \imath=f, c,
$$

i.e., they are exponential functions of the mean-field integral of the normalized vegetation density, $b\left(\mathbf{r}+\mathbf{r}^{\prime}, t\right)$, weighted by the kernels, $\Phi_{\mathrm{f}}$ and $\Phi_{\mathrm{c}}$, which describe the spatial extension of feedback effects in terms of the characteristic ranges $L_{\mathrm{f}}$ and $L_{\mathrm{c}}$ over which facilitative and competitive interactions operate. The positive parameters $\chi_{\mathrm{f}}$ and $\chi_{\mathrm{c}}$, which fix the strength of the interactions, may be influenced by extrinsic factors such as the degree of environmental aridity.

(iii) Our aim is to study the localized structures having the generic characteristic that they appear at the start to be a uniform density vegetation cover, or, more exactly, uniform stationary state solutions of (1) for which $b(\mathbf{r}, t)=b$ is a finite, positive constant, excluding the neighborhood of the value zero. In this respect, we may, for simplicity, consider that $L_{\mathrm{f}}$ and $L_{\mathrm{c}}$ are constants, ${ }^{2}$ and $\operatorname{set} L_{\mathrm{f}} \equiv L_{\mathrm{f}}^{0}=$ $L_{\mathrm{a}}$ and $L_{\mathrm{c}} \equiv L_{\mathrm{c}}^{0}=L_{\mathrm{r}}$. If we furthermore specify, in agreement with conditions (i)

\footnotetext{
${ }^{2}$ Clearly, $L_{\mathrm{f}}$ and $L_{\mathrm{c}}$ depend on the stage of development of the vegetation: mature plants, obviously, affect a greater territory than young seedlings. This feature plays an important role in phyto-societal behaviors which generically appear only at low average vegetation densities, i.e., in the neighborhood of $b=0$. The method consisting to link the development of the vegetation to its density by assuming that $L=L^{0} b\left(\mathbf{r}+\mathbf{r}^{\prime}, t\right)^{p}$, where $L^{0}$ is a constant and $p$ is an allometric exponent, constitutes a straightforward ansatz for handling such situation. Vegetation patterns consisting of vegetation patches distributed on bare soil can clearly be expected to be of this type. We shall report on them elsewhere [26].
} 
and (ii), that the interactions obey a gaussian, isotropic distribution law, ${ }^{3}$ then we obtain the following expressions (see Fig. 2) for the kernels $\Phi_{\mathrm{f}}$ and $\Phi_{\mathrm{c}}$ :

$$
\Phi_{l}\left(\left|\mathbf{r}^{\prime}\right|\right)=\mathscr{N}_{\iota} \exp ^{\left[-\frac{\left|\mathbf{r}^{\prime}\right|^{2}}{L_{l}^{0}}\right]}, \quad \text { with } \quad l=f, c, \quad L_{\mathrm{f}}^{0}=L_{\mathrm{a}}, L_{\mathrm{c}}^{0}=L_{\mathrm{r}} .
$$

The normalization condition

$$
\mathscr{N}_{l}=\frac{1}{\pi} \int \exp \left(-\frac{|\mathbf{r}|^{2}}{L_{l}^{0}}\right) \mathrm{d} \mathbf{r}
$$

must be imposed so that bulk behaviors, e.g., the uniform stationary solutions of (1), are independent of the interaction ranges in the case of spatially isotropic environments, as expected on physical grounds.

Setting the unit of length to be equal to the crown radius, $L_{\mathrm{a}}$, assuming that dispersion obeys a gaussian law, denoting the corresponding dispersion range of seeds (gaussian variance) by $L_{\mathrm{d}}$ and replacing (2),(3),(4),(5) and (6) in (1), the mean-field evolution equation of the vegetation finally reads

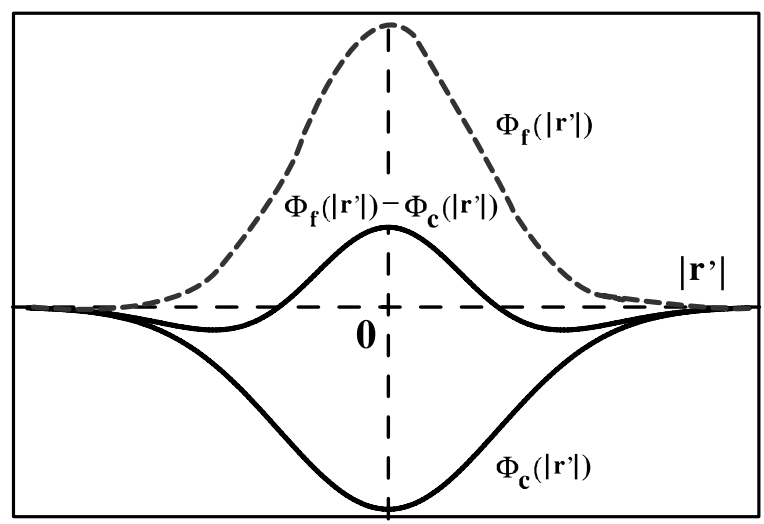

Fig. 2 Sketch of the facilitative and competitive mean-field kernels $\Phi_{\mathrm{f}}$ and $\Phi_{\mathrm{c}}$, as given by (5). Clearly, at short distances, $\left|\mathbf{r}^{\prime}\right|$, the difference $\Lambda=\Phi_{\mathrm{f}}-\Phi_{\mathrm{c}}$, which represents the effective meanfield generated by the interactions, is facilitative (positive values) while at great distances, it is competitive (negative values). The unit of length is equal to the canopy radius, $L_{\mathrm{a}}=1$

\footnotetext{
${ }^{3}$ From a qualitative point of view, this gaussian choice is convenient and implies no loss of generality. Other expressions for the kernels, $\Phi_{\mathrm{f}}$ and $\Phi_{\mathrm{c}}$, quantitatively describing specific vegetation systems, will be considered elsewhere [26]. Anyway, we are not interested here in the influence of external anisotropies. Hence, we have the choice of isotropic kernels, i.e., those that have no angular dependency and are only functions of the distance between points. For investigations devoted to the role of spatial anisotropies, see [3, 27, 28].
} 


$$
\begin{aligned}
\partial_{t} b(\mathbf{r}, t)= & b(\mathbf{r}, t)[1-b(\mathbf{r}, t)] \exp \left(\frac{\chi_{\mathrm{f}}}{\pi} \int \mathrm{e}^{-\left|\mathbf{r}^{\prime}\right|^{2}} b\left(\mathbf{r}+\mathbf{r}^{\prime}, t\right) \mathrm{d} \mathbf{r}^{\prime}\right) \\
& -\mu b(\mathbf{r}, t) \exp \left(\varepsilon \frac{\chi_{\mathrm{c}}}{\pi} \int \mathrm{e}^{-\varepsilon\left|\mathbf{r}^{\prime}\right|^{2}} b\left(\mathbf{r}+\mathbf{r}^{\prime}, t\right) \mathrm{d} \mathbf{r}^{\prime}\right) \\
& +\frac{\sigma D}{\pi} \int \mathrm{e}^{\sigma\left|\mathbf{r}^{\prime}\right|^{2}}\left[b\left(\mathbf{r}+\mathbf{r}^{\prime}, t\right)-b(\mathbf{r}, t)\right] \mathrm{d} \mathbf{r}^{\prime},
\end{aligned}
$$

where the parameters

$$
\varepsilon=\left(\frac{L_{\mathrm{a}}}{L_{\mathrm{r}}}\right)^{2} \quad \text { and } \quad \sigma=\left(\frac{L_{\mathrm{a}}}{L_{\mathrm{d}}}\right)^{2},
$$

specify the structural ratio and dispersivity of the vegetation considered.

In order to investigate the spatio-temporal dynamics predicted by the integrodifferential (7), it is convenient to work with a partial differential equation approximation of it. The latter is derived in the next section.

\section{Weak-Gradient Approximation}

We look for an approximation to (7), in the form of a partial differential equation, in the limit where, according to linear stability theory, (i) unstable fluctuations are of much greater wavelength than the crown size, $L_{\mathrm{a}}$, (ii) their growth rate is small, and (iii) nonlinearities remain weak. Generically, requiring (i) and (ii) means that we are interested in the system's dynamics near a transition point where fluctuations corresponding to the zero-Fourier mode become unstable, i.e., fluctuations of very large wavelength are stable but are nearly marginal (in the weak-gradient approximation). Requiring (iii) means that we look for conditions such that this transition point occurs at uniform vegetation densities, $b$, thus satisfying the inequalities $0<b<<1$. Hence, let us first determine the uniform stationary states of (7) and their stability properties. It is easily found that the latter, given by the solutions of

$$
\mu=b(1-b) \exp (\Lambda b)
$$

depends on only two parameters. The switching parameter $\mu$ (defined in (2)) controls the stability of the trivial uniform solution, $b=b_{0}=0$, corresponding to bare soil without vegetation: $b_{0}$ is unstable for $0 \leq \mu<1$ and stable for $\mu \geq 1$. The second parameter in (9) is the feedback balance resulting from the difference between the strengths of facilitative and competitive feedbacks:

$$
\Lambda=\chi_{\mathrm{f}}-\chi_{\mathrm{c}}
$$

It controls whether the population behaves co-operatively $(\Lambda>0)$ or not $(\Lambda<0)$. At the "neutral feedback point", $\Lambda=0$, facilitative and competitive effects balance each other exactly - the non-trivial uniform stationary state solution of (3), 


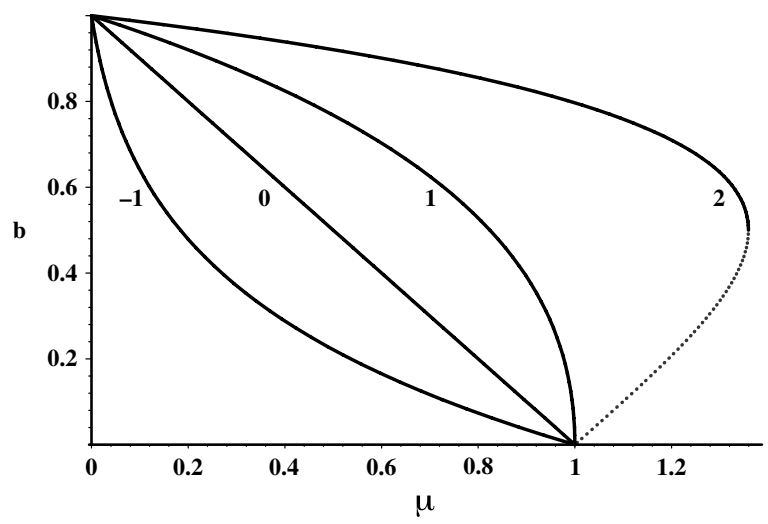

Fig. 3 Uniform stationary states solutions of (9) as a function of the switching parameter, $\mu$, with increasing values of the feedback balance, $\Lambda$. For $\Lambda>1$, the system exhibits a saddle-node transition point, whose co-ordinates are given by (11)

$b=b_{\mathrm{s}}=1-\mu$, is then identical to that of a community in which plants do not interact, i.e., one with $\chi_{\mathrm{f}}=\chi_{\mathrm{c}}=0$. For $\Lambda>1$ and $1 \leq \mu \leq \exp (\Lambda-1) / \Lambda$, (9) admits three non-negative solutions, $b_{0}, b_{-}, b_{+}$(see Fig. 3 ). The uniform state, $b_{-}$, is always unstable. On the other hand, $b_{0}$ and $b_{+}$are stable with respect to small perturbations which do not break the system spatial uniformity. Remarkably, when the feedback balance, $\Lambda$, is strongly co-operative, i.e., for $\Lambda>>1$, the domain of existence of the vegetation, represented by the $b_{+}$branch of stationary states, extends up to the saddle-node transition point of co-ordinates

$$
b^{*}=\frac{\Lambda-1}{\Lambda}, \quad \mu^{*}=\frac{\exp (\Lambda-1)}{\Lambda},
$$

i.e., far beyond the switching point, $\mu=1$, where the trivial state, $b_{0}$, changes stability. In light of the requirements (i) - (iii) mentioned earlier, we now explore the system dynamics in the neighborhood of the feedback balance value $\Lambda=1$. Hence, we set

$$
b(\mathbf{r}, t)=\zeta u(\mathbf{r}, t), \quad \Lambda=1+\zeta \Lambda_{1}, \quad \mu=1+\zeta^{2} \mu_{2},
$$

where $\zeta$ is a "smallness" parameter, and we renormalize the time and space scales by the transformation:

$$
t=\frac{2 \tilde{t}}{\zeta^{2}}, \quad|\mathbf{r}|=\frac{|\widetilde{\mathbf{r}}|}{k_{0}},
$$

where $k_{0}$ is the non-zero, positive modulus of the first Fourier wave vector which becomes (linearly) unstable in the neighborhood of the saddle-node point. (For simplicity of notation, the tilde will later be dropped.) We require $k_{0}<<1$, i.e., that it corresponds to spatial heterogeneities of much greater size than $L_{\mathrm{a}}$. The linear stability analysis of (7) shows that, in order to satisfy this condition and to obtain real, positive values for $k_{0}$, the strength of competitive feedbacks, $\chi_{\mathrm{c}}$, and the dispersion coefficient, $D$, must scale with respect to $\zeta$ as: 


$$
\chi_{\mathrm{c}}=\frac{\varepsilon}{1-\varepsilon}+\sqrt{\zeta} \chi_{1}, \quad D=\zeta^{3 / 2} \frac{\delta}{\sigma} .
$$

To the dominant order in $\zeta$, this yields

$$
k_{0}=q \zeta^{1 / 4}, \quad \text { with } \quad q=2 \sqrt{\varepsilon\left(\chi_{1}-2 \frac{\delta}{u_{+}}\right)}
$$

where

$$
u_{+}=\Lambda_{1}+\sqrt{\Lambda_{1}^{2}-2 \mu_{2}}
$$

is the dominant term in the expansion $b_{+}=\zeta u_{+}+\mathscr{O}\left(\zeta^{2}\right)$ of the branch of stationary states, $b_{+}$, in terms of $\zeta$.

We use (10) to eliminate $\chi_{\mathrm{f}}$, expand $b\left(\mathbf{r}+\mathbf{r}^{\prime}, t\right)=\zeta u\left(\mathbf{r}+\mathbf{r}^{\prime}, t\right)+\mathscr{O}\left(\zeta^{2}\right)$ in a Taylor series around the focal point $\mathbf{r}$, substitute (12), (13), (14), and (15) into (7) and expand the resulting equation in terms of $\zeta$, up to $\mathscr{O}\left(\zeta^{3}\right)$. This yields the desired partial differential evolution equation, with $u(\mathbf{r}, t)$ as new state variable:

$$
\begin{aligned}
\partial_{t} u(\mathbf{r}, t)= & -u(\mathbf{r}, t)\left[2 \mu_{2}-2 \Lambda_{1} u(\mathbf{r}, t)+u(\mathbf{r}, t)^{2}\right] \\
& +\left[q \delta-\frac{q \chi_{1}}{2} u(\mathbf{r}, t)\right] \nabla^{2} u(\mathbf{r}, t)-\frac{q^{2}}{16 \varepsilon} u(\mathbf{r}, t) \nabla^{4} u(\mathbf{r}, t) \\
& +\mathscr{O}\left(\zeta^{7 / 2}\right) .
\end{aligned}
$$

In order to put (17) into a more compact form, let us define

$$
\eta=2 \mu_{2} \quad \kappa=2 \Lambda_{1}, \quad \Delta=q \delta, \quad \Gamma=\frac{q \chi_{1}}{2}, \quad \alpha=\frac{q^{2}}{16 \varepsilon} .
$$

Rewritten in terms of these parameters, (17) becomes

$$
\begin{aligned}
\partial_{t} u(\mathbf{r}, t)= & -u(\mathbf{r}, t)\left[\eta-\kappa u(\mathbf{r}, t)+u(\mathbf{r}, t)^{2}\right] \\
& +[\Delta-\Gamma u(\mathbf{r}, t)] \nabla^{2} u(\mathbf{r}, t)-\alpha u(\mathbf{r}, t) \nabla^{4} u(\mathbf{r}, t),
\end{aligned}
$$

while the stationary solutions, representing uniform vegetation covers, are now given by

$$
u_{0}=0, \quad u_{ \pm}=\left[\kappa \pm \sqrt{\kappa^{2}-4 \eta}\right] / 2
$$

To be physically acceptable, $u_{ \pm}$must be real and non-negative. Two situations must be distinguished, according to the sign of $\kappa$. When $\kappa<0$, the co-ordinates of the saddle-node point are unphysical. Besides $u_{0}, u_{+}$is then the only acceptable uniform stationary state solution. It exists only if $\eta<0$ and is a monotonically decreasing function of $\eta$ which vanishes for $\eta=0$. When $\kappa>0$, the co-ordinates of the saddlenode point, given by

$$
u^{*}=\kappa / 2, \quad \eta^{*}=\kappa^{2} / 4,
$$


are physically acceptable. Accordingly, for $0<\eta<\eta^{*}$, the bio-mass density exhibits a phenomenon of bistability, so that $u_{0}$ and $u_{+}$, which are linearly stable with respect to uniform fluctuations, as well as the intermediate unstable branch, $u_{-}$, are then, simultaneously, acceptable uniform solutions of (19). In the following, we shall focus on the parameter regime where the uniform plant distribution exhibits bistability $(\kappa>0)$, and assume that the inequalities $\left(\eta_{\mathrm{m}}<\eta<\eta^{*}\right)$ hold. In that case, all uniform stationary states belonging to $u_{+}$have a finite, non-negative value. This is required, given the simplification made earlier that $L_{\mathrm{f}}$ and $L_{\mathrm{c}}$ are constants, independent of the vegetation density (cf. assumption (iii) in 2).

\section{Clustering and Periodic Vegetation Patterns}

Inspection of (19) suggests that the $u_{+}$stationary states may become unstable if subjected to random, non-uniform fluctuations. Indeed, when $\Delta / \Gamma=2 \delta / \chi_{1}<u_{+}$, the "diffusion" coefficient in front of the Laplacian is negative, indicating that vegetation clustering, i.e., the "phase separation" of vegetation and bare soil, becomes possible [29, 30]. This phenomenon is physically comparable with the behavior of immiscible mixtures, e.g., the separation of oil and water. It corresponds, in the linear stability analysis of (19), to the appearance of a finite band of unstable Fourier modes. Its upper cutoff is due to the "line tension" coefficient of the bi-Laplacian term, which is always stabilizing (for short distances, dispersion is always an efficient mixing mechanism), while its lower cutoff owes its existence to the stability of the zero-Fourier mode. (By definition, all $u_{+}$stationary states are stable with respect to uniform fluctuations.) Under these conditions, the intrinsic instability due to the negative diffusion coefficient is modulational - random noise triggers the appearance of spatially periodic patterns which spread over the whole territory. The wavelength of the first non-zero-Fourier mode to become unstable is

$$
\lambda_{\mathrm{m}}=2 \pi \sqrt{\frac{2 \alpha}{\Gamma / \alpha-\Delta / u_{\mathrm{m}}}},
$$

where $u_{\mathrm{m}}$, given by the largest real positive solutions of the cubic polynomial

$$
\left(2 \Gamma u_{\mathrm{m}}-\Delta\right)^{2}=4 \alpha u_{\mathrm{m}}^{2}\left(2 u_{\mathrm{m}}-\alpha\right),
$$

is the threshold state at which the modulational instability appears on the $u_{+}$branch of solutions for $\eta=\eta_{\mathrm{m}}$ (see Fig. 4). When the aridity parameter, $\eta$, increases, the structures that appear first (sub-critically) are the so-called hexagons, H0. They consist of a periodic pattern, of hexagonal symmetry, made up of sparse vegetation spots. Upon a further increases in $\eta$, this pattern becomes unstable and the system evolves toward patterns consisting of bands or stripes; the latter properties are in good agreement with observations relating to tiger bush patterns. Increasing aridity still further destabilizes these banded patterns and transforms them into 


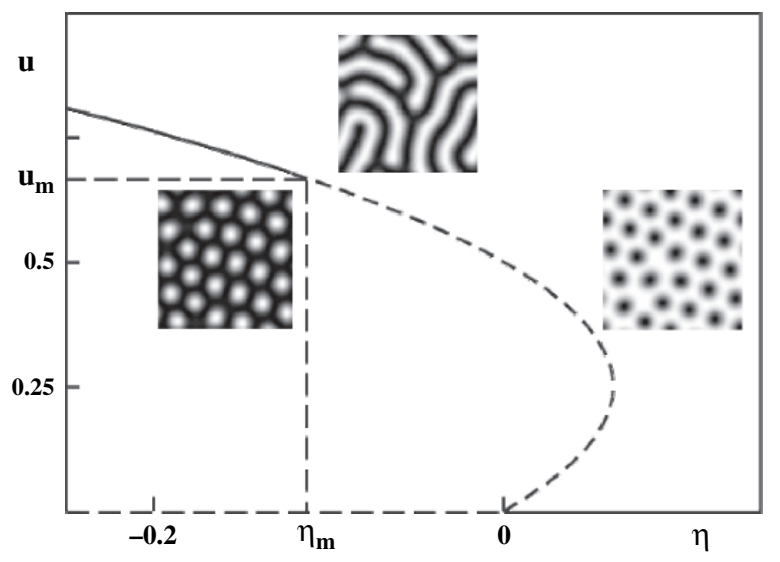

Fig. 4 Uniform stationary bio-mass density as a function of the aridity parameter $\eta$, for $\kappa=0.5$, $\Delta=0.1, \Gamma=0.5$ and $\alpha=0.25$. Broken lines correspond to unstable states. As $\eta$ increases, at the bifurcation point $\left(\eta_{\mathrm{m}}, u_{\mathrm{m}}\right)$, the uniform branch of stationary states, $u_{+}$, becomes unstable with respect to infinitesimal fluctuations whose wavelength is given by (22). In the neighborhood of this point, sub-critical periodic vegetation patterns which are made up of spots of sparser vegetation can be found. Super-critically, they transform into stripes, and finally into periodic patterns consisting of vegetation spots separated by bare ground. The three different kinds of patterns represented have been obtained for $\eta=-0.25,0.1$ and 0.2 , respectively. The grid is $128 \times 128$ points. Black corresponds to the highest values of the bio-mass density. Minima are plain white. Periodic boundary conditions are used in both spatial co-ordinates

hexagonal $\mathrm{H} \pi$ patterns which correspond to periodic distributions of vegetation spots surrounded by bare ground. These three types of $2 \mathrm{D}$ periodic structures are represented in Fig. 4. They have been obtained by numerically integrating (19) on a square-shaped domain with periodic boundary conditions. The initial condition used to generate these structures consists of the unstable uniform stationary solution, perturbed by noise of small amplitude. Reversing the variation of the aridity parameter shows that there exist two hysteresis loops involving striped, $\mathrm{H} 0$ and $\mathrm{H} \pi$ patterns (not represented). Published works giving nonlinear analyses $[13,31]$ allow for detailed calculation of the bifurcation diagrams of such periodic vegetation patterns.

\section{Pinning and Localized Bare Soil Spots}

Localized vegetation patterns have been described in various arid regions, notably in Africa. So far, however, they have attracted little attention, both from experimental and theoretical points of view. Such patterns are "spatially localized", in the sense that they are elemental structures (vegetation patches or holes in the vegetation cover) which have a well-defined size and which seem to be quite stable. However, in contrast to periodic patterns, they apparently have little or no tendency 
to spread and to invade the whole territory accessible to them. Characteristically, they are found as isolated elements, or in the form of groups which gather a small number of elements, more or less closely. The fairy circles illustrated in Fig. 1 are striking examples belonging to this category of patterns.

On the theoretical side, spatial localization is a patterning phenomenon better known in contexts of a physico-chemical rather than biological nature. It is established that various, quite distinct, non-equilibrium reaction-diffusion systems and nonlinear optical devices, which have the common property that they exhibit modulational instability, also display pinning phenomena which generate so-called localized structures (LS) [32, 33, 34, 35, 36, 37, 38, 39, 40, 41]. In all these systems, the conditions under which periodic patterns and localized structures appear are closely related - dynamically speaking, in all cases, a sub-critical modulational instability underlies the pinning phenomena responsible for the appearance of localized patterns. In this section, (i) we show that the dynamics described by (19) gives rise to such pinning phenomena and, as a consequence, may account for the formation of the localized bare spots (LBS) observed, e.g., the case of fairy circles; and (ii) we present a treatment which allows us to analytically evaluate the elemental solutions of (19) corresponding to an isolated bare soil spot immersed in an otherwise uniform vegetation cover.

Mathematically, stable localized structures are homoclinic solutions (solitary waves or stationary pulses) which belong to the sub-critical domain where a uniform branch of stationary state solutions and a branch of spatially periodic solutions are both linearly stable [34]. In parameter space, this situation corresponds to the existence of a hysteresis loop. Within it, there generally exists a so-called pinning range of parameter values for which stable localized structures, connecting the uniform and the periodic solutions, can be found. Their stability is attributed to the absence of a variational principle, i.e., to the non-existence of a Lyapunov functional guaranteeing that evolution proceeds toward the state for which the functional has the smallest possible value which is compatible with the system boundary conditions. The Swift-Hohenberg equation, for example, is a paradigmatic evolution equation which admits localized structures of this kind. ${ }^{4}$ Clearly, the presence of the nonlinear diffusion terms, $u \nabla^{2} u$ and $u \nabla^{4} u$, render (19) non-variational.

Figure 5 shows some examples of localized structures obtained by numerically solving (19) for given, fixed values of the control parameters. Because of the homoclinic nature of these solutions, the number, as well as the spatial location, of the bare spots immersed in the bulk of the linearly stable uniform reference state depends on the initial condition considered. A single, isolated stationary bare spot is shown in Fig. 5(a). The spatial profile of the bio-mass density surrounding the central bare spot exhibits a decaying spatial oscillation which produces the concentric rings seen in the Fig. 5 (see also Fig. 7). This oscillatory tail connects the uniformly vegetated state to the central spot of sparser vegetation. To a first approximation, its characteristics (wavelength and maximal amplitude) are those ofthe periodic solution

\footnotetext{
${ }^{4}$ It is noteworthy that LS do not require a commutation process between distinct uniform stationary states [35].
} 

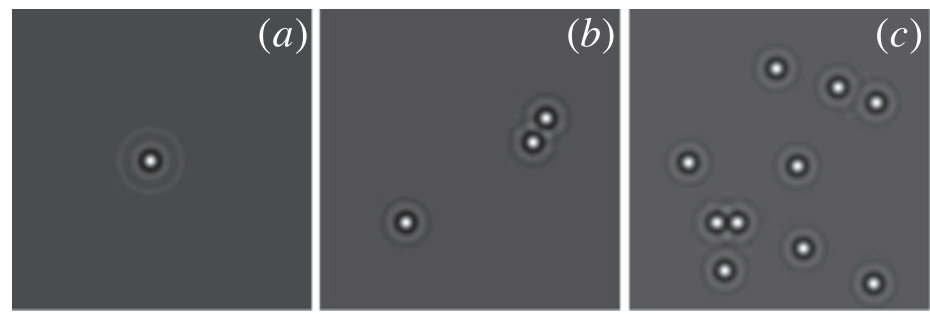

Fig. 5 Sample of localized bare spots surrounded by the uniformly vegetated state $u_{\mathrm{S}}=(\kappa+$ $\left.\sqrt{\kappa^{2}-4 \eta}\right) / 2$, obtained from numerical simulations of the model (19). Parameters are $\kappa=0.5$, $\Delta=0.1, \Gamma=2 \alpha=0.5$ and $\eta=-0.025$. (a) Single bare spot, (b) three LBSs (c) random distribution of LBS. The grid is $256 \times 256$ points. Black corresponds to the highest values of the phyto-mass density. Minima are plain white. Periodic boundary conditions are used in both spatial co-ordinates

corresponding to the same parameter values. Figure 5(b) shows a pattern consisting of three localized bare spots, of which one is an isolated structure and two form a bound state. In Fig. 5(c), a pattern consisting of randomly distributed isolated and bound bare spots has been obtained.

Amplitude equations describing the space-time evolution of a slow unstable mode, derived in the framework of weakly-nonlinear regime, cannot describe localized structures. This approach does not take into account the non-adiabatic effects that involve the fast spatial scales which are responsible for the stabilization of LSs [33]. In recent years, considerable progress has been realized in the understanding of these structures. Analytical localized structures are relatively well understood in the 1D setting. The existence of localized structures in the Swift-Hohenberg model has been found by using functional analysis methods [42] and a dynamical system approach [43, 44, 45]. More recently, the computation of small and large spatial scales, by going beyond all orders of the usual multiple-scale expansion, allows us to construct the bifurcation diagram of localized structures [46]. However, analytical methods in 2D are still largely unexplored, and most of the results are obtained by numerical simulations.

A single stationary bare spot solution of (19) can be written in the form $u(r)=$ $U(r)+u_{+}$, where $U(r)$ is the deviation from the uniform stationary state, $u_{+}$. The stability of the bare spot solution is determined by the spectrum of the linear operator $L$, defined by

$$
L\left[U(r)+u_{+}\right]=L\left(u_{+}\right)+\mathscr{M}\left[u_{+}, U(r)\right] U(r),
$$

with

$$
\begin{aligned}
L\left(u_{+}\right) & =-\eta+2 \kappa u_{+}-3 u_{+}^{2}+\left(\Delta-\Gamma u_{+}\right) \nabla^{2}-\alpha u_{+} \nabla^{4}, \\
\mathscr{M}\left[u_{+}, U(r)\right] & =\left(\kappa-3 u_{+}\right)-U(r)-\Gamma \nabla^{2}-\alpha \nabla^{4} .
\end{aligned}
$$


A single LBS is a radially symmetric solution, $u(x, y)=u(r)$, of (19). We can then replace the Laplacian operator by $\nabla^{2}=\partial_{r r}^{2}+(1 / r) \partial_{r}$. We consider the following boundary conditions: $\left.u\right|_{r=+\infty}=u_{+},\left.\partial_{r} u\right|_{r=0}=0,\left.\partial_{r} u\right|_{r=+\infty}=0$ and $\left.\partial_{r}^{3} u\right|_{r=0}=0$. The solution of the linear problem is

$$
u(r)=u_{+}+\Re\left\{A \exp (\mathrm{i} \theta) K_{0}[(\varpi+\mathrm{i} \omega) r]\right\},
$$

where

$$
\varpi+\mathrm{i} \omega=\sqrt{-\xi_{1}+\mathrm{i} \xi_{2}}
$$

with

$$
\left.\xi_{1}=\left[\left(\Delta / u_{+}\right)-\Gamma\right)\right] /(2 \alpha), \quad \text { and } \quad \xi_{2}=\sqrt{\left(3 u_{+}^{2}+\eta-2 \kappa u_{+}\right) /\left(\alpha u_{+}\right)-\xi_{1}^{2}} .
$$

The Bessel function $K_{0}$ describes the decaying oscillations at large distance from the center of the bare spot. The bare spots are calculated in the following manner. First, we integrate (19) with $\partial_{\mathrm{t}} u=0$, from $r=10^{-8}$ to $r_{1}=1$, using the initial conditions: $\left.u(r)\right|_{r=r_{0}}=B,\left.\partial_{r}^{2} u\right|_{r=r_{0}}=C$ and $\left.\partial_{r} u\right|_{r=r_{0}}=\left.\partial_{r}^{3} u\right|_{r=r_{0}}=0$. Then we solve the (19) with $\partial_{t} u=0$ from $r=r_{1}$ to $r=L$, with $L=100$ for the initial condition of (26). The parameters $A, B, C$ and $\theta$ are determined numerically by matching the solution obtained at $r=r_{1}$. The procedure of the calculation is the shooting method. This calculation allows us to draw the bifurcation diagram (see Fig. 6), where we plot the homogeneous steady state together with the minimum values of the bare spot. The spot branch of solutions emerges from the uniformly vegetated state, $u_{+}$, at the bifurcation point $\left(\eta_{\mathrm{m}}, u_{\mathrm{m}}\right)$ associated with the modulational instability.

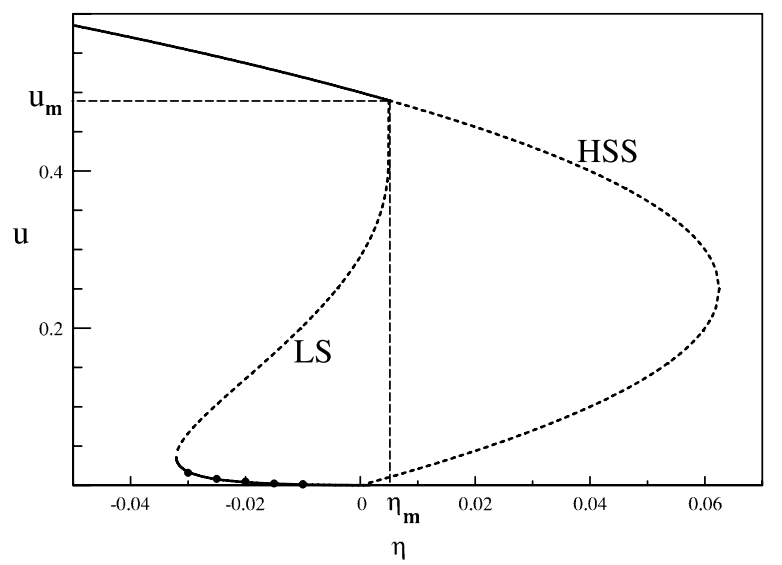

Fig. 6 Bifurcation diagram. The solid (dotted) line represents the stable (unstable) solutions. As the aridity parameter increases, the upper uniformly vegetated state (HSS) becomes unstable at the bifurcation point $\left(\mu_{\mathrm{m}}, u_{\mathrm{m}}\right)$. From this instability point, a branch of localized bare spots (BLS) emerges sub-critically. Here $\kappa=0.5, \Delta=0.1, \Gamma=2 \alpha=0.5$, and $\eta=-0.025$. The black circles indicate the minimum of LBS obtained from the numerical simulations of the model (19). The agreement between the two method is excellent 


\section{Attractive/Repulsive Interactions Between Bare Spots}

We have seen that (19) admits bare spot solutions that either can be spatially independent, or, if they are close enough, can interact through their overlapping tails to form bound states. In this section, we study the properties of these bound states in the case of two LBSs interacting in 2D space. An analytical expression, in terms of integrals of Bessel functions, is derived for the interaction forces between two separate localized bare spots. The interaction will initiate motion of the LBSs until they reach a stable equilibrium position. We show that there exist several equilibrium positions and that the system selects one of these states, depending on the initial distance between the two localized spots. On the other hand, numerical simulations reveal that two localized bare spots may loose their stability under their mutual interaction, giving rise to a periodic distribution of bare spots forming an hexagonal lattice.

Figure 7 shows two situations which are representative of the behaviors which may be observed when two LBSs interact. In order to analyze them, let us first rewrite (19) as:
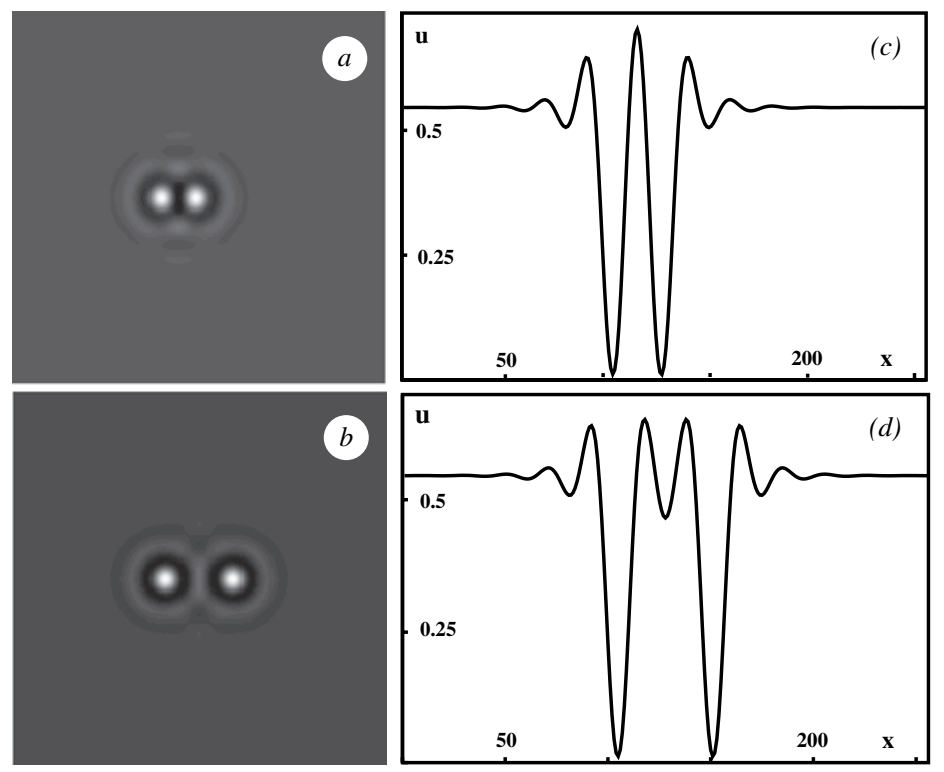

Fig. 7 Interaction between two LBSs. (a) and (b) are obtained for the same values of parameters. They differ only in the initial distance between the LBSs. (c) and (d) are, respectively, the crosssections taken from (a) and (b) and passing through the center, along the $x$-direction. Parameters are $\kappa=0.5, \Delta=0.1, \Gamma=2 \alpha=0.5$ and $\eta=-0.025$. The grid is $256 \times 256$ points. Black corresponds to the highest values of the phyto-mass density. Minima are plain white. Periodic boundary conditions are used in both spatial co-ordinates 


$$
\begin{aligned}
\partial_{t} u & =F[u], \\
F(u) & =\Delta \nabla^{2} u-\eta u+\kappa u^{2}-u^{3}-u\left(\Gamma \nabla^{2} u+\alpha \nabla^{4} u\right) .
\end{aligned}
$$

In the following, we consider two bare spots that can interact through their oscillatory tails. The asymptotic form of the tail is described by a modified Bessel function (26) that decays at large distances from the centre of the localized bare spot. When they are excited sufficiently close to each another, they exert mutual forces due to the overlapping of their tails. We will see that these interaction forces can be either attractive or repulsive, depending on the initial distance between the bare spots. In the following, we calculate these interaction forces analytically for the case of weak overlap, which means that the two bare spots are well separated.

Let us choose the co-ordinate system in such a way that its origin is located at the mid-point between two spots, i.e., at equal distance from each of them. Let the center of mass of each of the bare spots be on the axis $x$, so that their minima are located at the points $(-R / 2,0)$ and $(0, R / 2)$ along the $x$-direction, where $R$ is the distance between them (Fig. 8). We look for a solution of (19) in the form of a slightly perturbed linear superposition of two spots:

$$
U(\mathbf{r}, t)=U_{1}(\mathbf{r})+U_{2}(\mathbf{r})+\varepsilon \delta U(\mathbf{r}, t) \quad \text { where } \quad U_{1,2}(\mathbf{r})=U\left(\left|\mathbf{r}-\mathbf{R}_{1,2}\right|\right) .
$$

The positions of the localized solutions, $\mathbf{R}_{1,2}(t)=\left(X_{1,2}, Y_{1,2}\right)^{\mathrm{T}}$, evolve on the slow time scale $\partial_{t} \mathbf{R}_{1,2}(t)=\mathscr{O}(\varepsilon)$. Substituting this expansion into (19) and collecting first-order terms in $\varepsilon$, we obtain

$$
\left[\partial_{t}-L\left(U_{1}+U_{2}+u_{+}\right)\right] \delta U=\nabla U_{1} \cdot \partial_{t} \mathbf{R}_{1}+\nabla U_{2} \cdot \partial_{t} \mathbf{R}_{2}+F\left(U_{1}+U_{2}+u_{+}\right) .
$$

To solve (31), it is necessary to satisfy a solvability condition, viz. the right side of (31) should be orthogonal to the null eigenfunctions of the adjoint operator $L^{\dagger}\left(U_{1}+U_{2}+u_{+}\right)$, which can be approximated by the neutral (often called Goldstone) translational modes, $\mathbf{v}_{1}=\left(v_{1 x}, v_{1 y}\right)^{\mathrm{T}}$ and $\mathbf{v}_{2}=\left(v_{2 x}, v_{2 y}\right)^{\mathrm{T}}$ of $L^{\dagger}\left(U_{1}+u_{+}\right)$ and $L^{\dagger}\left(U_{2}+u_{+}\right)$, respectively. The solvability condition reads

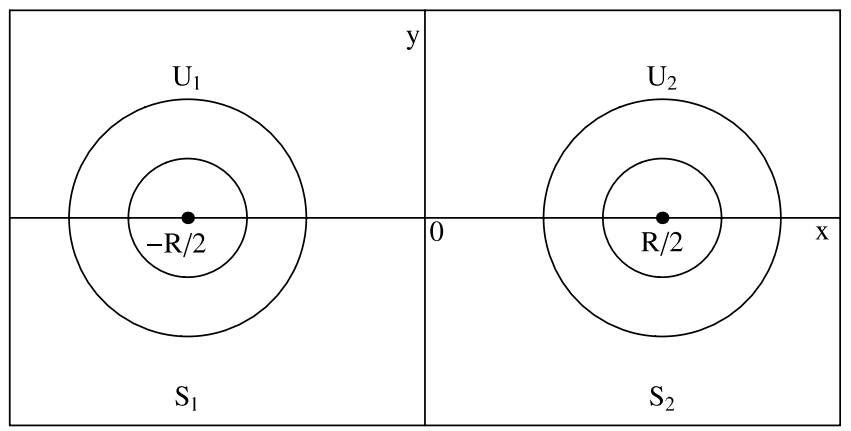

Fig. 8 Schematic plot of two bare spots located at the points $(-R / 2,0)$ and $(0, R / 2)$ along the $x$-direction. The black circles indicate the tails of the LBSs 


$$
\frac{1}{2} \partial_{t} \mathbf{R}_{1} \int_{S} \mathbf{v}_{k} \cdot \nabla U_{1} \mathrm{~d} \mathbf{r}+\frac{1}{2} \partial_{t} \mathbf{R}_{2} \int_{S} \mathbf{v}_{k} \cdot \nabla U_{2} \mathrm{~d} \mathbf{r}=-\int_{S} \mathbf{v}_{k} F\left(U_{1}+U_{2}+u_{+}\right) \mathrm{d} \mathbf{r},
$$

with $k=1,2$. The integrals appearing in (32) are taken over the whole plane $S=$ $\mathfrak{R} \times \mathfrak{R} \equiv(x, y)$. To perform the integration over this plane, we decompose it into two half planes, namely, $S_{1}=\mathfrak{R}^{-} \times \mathfrak{R}$ and $S_{2}=\mathfrak{R}^{+} \times \mathfrak{R}$ (see Fig. 8)

$$
\begin{aligned}
\int_{S} \mathbf{v}_{k} F\left(U_{1}+U_{2}+u_{+}\right) \mathrm{d} \mathbf{r}= & \int_{S_{1}} \mathbf{v}_{k} F\left(U_{1}+U_{2}+u_{+}\right) \mathrm{d} \mathbf{r} \\
& +\int_{S_{2}} \mathbf{v}_{k} F\left(U_{1}+U_{2}+u_{+}\right) \mathrm{d} \mathbf{r} .
\end{aligned}
$$

In the half plane $S_{1}$, where the bare spot solution $U_{2}$ is small, we can apply the following first-order expansion:

$$
F\left(U_{1}+U_{2}+u_{+}\right) \approx F\left(U_{1}+u_{+}\right)+L\left(U_{1}+u_{+}\right) U_{2}=L\left(U_{1}+u_{+}\right) U_{2} .
$$

Similarly, in the half-plane $S_{2}$, where the bare spot solution $U_{1}$ is small,

$$
F\left(U_{1}+U_{2}+u_{+}\right) \approx F\left(U_{2}+u_{+}\right)+L\left(U_{2}+u_{+}\right) U_{1}=L\left(U_{2}+u_{+}\right) U_{1} .
$$

Then (32) reads

$$
\xi \partial_{t} \mathbf{R}_{1}=-\int_{S_{1}} \mathbf{v}_{1} L^{\dagger}\left(U_{1}+u_{+}\right) U_{2} \mathrm{~d} \mathbf{r}
$$

and

$$
\xi \partial_{t} \mathbf{R}_{2}=-\int_{S_{2}} \mathbf{v}_{2} L^{\dagger}\left(U_{2}+u_{+}\right) U_{1} \mathrm{~d} \mathbf{r}
$$

with

$$
\xi=\frac{1}{2} \int_{S} \mathbf{v}_{1} \cdot \nabla U_{1} \mathrm{~d} \mathbf{r}=\frac{1}{2} \int_{S} \mathbf{v}_{2} \cdot \nabla U_{2} \mathrm{~d} \mathbf{r} .
$$

Subtracting (36) from (37), we get the equation for the time evolution of the distance between the spots:

$$
\xi \partial_{t} \mathbf{R}=-\int_{S_{2}} \mathbf{v}_{2} L^{\dagger}\left(U_{2}+u_{+}\right) U_{1} \mathrm{~d} \mathbf{r}+\int_{S_{1}} \mathbf{v}_{1} L^{\dagger}\left(U_{1}+u_{+}\right) U_{2} \mathrm{~d} \mathbf{r}
$$

where $\mathbf{R}=\mathbf{R}_{2}-\mathbf{R}_{1}$. Since $\mathbf{v}_{1,2}$ are the eigenfunctions of $L^{\dagger}\left(U_{1,2}+u_{+}\right)$with the zero eigenvalue, we have

$$
\begin{aligned}
\int_{S} U_{1} L^{\dagger}\left(U_{2}+u_{+}\right) \mathbf{v}_{2} \mathrm{~d} \mathbf{r} & =0=\int_{S} \mathbf{v}_{2} L\left(U_{2}+u_{+}\right) U_{1} \mathrm{~d} \mathbf{r} \\
& =\int_{S_{1}} \mathbf{v}_{2} L\left(U_{2}+u_{+}\right) U_{1} \mathrm{~d} \mathbf{r}+\int_{S_{2}} \mathbf{v}_{2} L\left(U_{2}+u_{+}\right) U_{1} \mathrm{~d} \mathbf{r}
\end{aligned}
$$




$$
\begin{aligned}
\int_{S} U_{2} L^{\dagger}\left(U_{1}+u_{+}\right) \mathbf{v}_{1} \mathrm{~d} \mathbf{r} & =0=\int_{S} \mathbf{v}_{1} L\left(U_{1}+u_{+}\right) U_{2} \mathrm{~d} \mathbf{r} \\
& =\int_{S_{1}} \mathbf{v}_{1} L\left(U_{1}+u_{+}\right) U_{2} \mathrm{~d} \mathbf{r}+\int_{S_{2}} \mathbf{v}_{1} L\left(U_{1}+u_{+}\right) U_{2} \mathrm{~d} \mathbf{r} .
\end{aligned}
$$

Using the relations (40) and (41), (39) becomes

$$
\xi \partial_{t} \mathbf{R}=\int_{S_{2}} \mathbf{v}_{1} L\left(U_{1}+u_{+}\right) U_{2} \mathrm{~d} \mathbf{r}-\int_{S_{1}} \mathbf{v}_{2} L\left(U_{2}+u_{+}\right) U_{1} \mathrm{~d} \mathbf{r} .
$$

Since $U_{1}\left(U_{2}\right)$ is small in $S_{2}\left(S_{1}\right)$, (42) can be approximated by

$$
\begin{aligned}
\xi \partial_{t} \mathbf{R}= & \int_{S_{2}}\left[\mathbf{v}_{1} L\left(u_{+}\right) U_{2}-U_{2} L\left(u_{+}\right) \mathbf{v}_{1}\right] \mathrm{d} \mathbf{r} \\
& -\int_{S_{1}}\left[\mathbf{v}_{2} L\left(u_{+}\right) U_{1}-U_{1} L\left(u_{+}\right) \mathbf{v}_{2}\right] \mathrm{d} \mathbf{r},
\end{aligned}
$$

where the self-adjoint linear operator $L\left(u_{+}\right)$is defined by (24) and $L\left(u_{+}\right) \mathbf{v}_{1}=O\left(\varepsilon^{2}\right)$ in $S_{2}$ while $L\left(u_{+}\right) \mathbf{v}_{2}=O\left(\varepsilon^{2}\right)$ in $S_{1}$. Using this equation, we get

$$
\xi \partial_{t} \mathbf{R}=\left(\Delta-\Gamma u_{+}\right)\left(\mathbf{I}_{1}-\mathbf{I}_{2}\right)+\alpha u_{+}\left(\mathbf{J}_{1}-\mathbf{J}_{2}\right)=\mathbf{F},
$$

with

$$
\begin{aligned}
& \mathbf{I}_{1,2}=\int_{S_{1,2}}\left[\mathbf{v}_{1,2} \nabla^{2} U_{2,1}-U_{2,1} \nabla^{2} \mathbf{v}_{1,2}\right] \mathrm{d} \mathbf{r}, \\
& \mathbf{J}_{1,2}=\int_{S_{1,2}}\left[\mathbf{v}_{1,2} \nabla^{4} U_{2,1}-U_{2,1} \nabla^{4} \mathbf{v}_{1,2}\right] \mathrm{d} \mathbf{r} .
\end{aligned}
$$

Using Green's identities and the symmetry properties $U(-r)=U(r)$ and $\mathbf{v}(-r)=$ $-\mathbf{v}(r)$, the integrals (45) and (46) over the half-planes $S_{k}, k=1,2$, can be transformed into the following integrals over the line $x=0$ separating these two halfplanes:

$$
\begin{aligned}
& \mathbf{I}_{k}=(-1)^{k} \int_{-\infty}^{\infty}\left[\frac{\mathrm{d}}{\mathrm{d} x}\left(\mathbf{v}_{k} U_{k}\right)\right]_{x=0} \mathrm{~d} y=-\nabla_{\mathbf{R}} \bar{I}_{k}, \\
& \mathbf{J}_{k}=2(-1)^{k} \int_{-\infty}^{\infty}\left[\frac{\mathrm{d}}{\mathrm{d} x}\left(U_{k} \nabla^{2} \mathbf{v}_{k}+\mathbf{v}_{k} \nabla^{2} U_{k}\right)\right]_{x=0} \mathrm{~d} y=-\nabla_{\mathbf{R}} \bar{J}_{k},
\end{aligned}
$$

where

$$
\begin{aligned}
& \bar{I}_{k}=2(-1)^{k} \int_{-\infty}^{\infty}\left(v_{k x} U_{k}\right)_{x=0} \mathrm{~d} y \\
& \bar{J}_{k}=4(-1)^{k} \int_{-\infty}^{\infty}\left(U_{k} \nabla^{2} v_{k x}+v_{k x} \nabla^{2} U_{k}\right)_{x=0} \mathrm{~d} y .
\end{aligned}
$$


According to (47) and (48), (44) can be rewritten in the following gradient form:

$$
\xi \partial_{t} \mathbf{R}=-\nabla_{\mathbf{R}} \mathscr{U}(R),
$$

with the potential function

$$
\mathscr{U}(R)=\left(\Delta-\Gamma u_{+}\right)\left(\bar{I}_{1}-\bar{I}_{2}\right)+\alpha u_{+}\left(\bar{J}_{1}-\bar{J}_{2}\right)
$$

Finally, we note that the integrals appearing in (45),(46),(47),(48),(49), and (50) have the symmetry properties $I_{2}=-I_{1}, J_{2}=-J_{1}, \bar{I}_{2}=-\bar{I}_{1}$ and $\bar{J}_{2}=-\bar{J}_{1}$, which express the fact that the velocities of the two spots have opposite signs, $\partial_{t} \mathbf{R}_{2}(t)=$ $-\partial_{t} \mathbf{R}_{1}(t)$.

In order to calculate the interaction force of two bare spots, we substitute the asymptotic relations for $U_{k}$ and $\mathbf{v}_{k}$, in terms of the modified Bessel functions, into (47) and (48) and perform the integration. The plot of the interaction force, $F$, versus the half-distance, $R / 2$, between the centers of the two bare spots is shown in Fig. 9. This function shows a decaying oscillation, as predicted from the linear analysis. The equilibrium positions $R_{1}, R_{2}$ and $R_{3}$ correspond to a separation where the interaction force between the two spots vanishes, i.e., $F=0$. The two spots are bound together by the interaction forces. The equilibrium positions $R_{1}$ and $R_{2}$ are stable, while $R_{3}$ is unstable. If the initial distance, $d$, between two spots is such that $R_{0}<d<R_{1}$, the system will evolve toward the formation of two spots separated by a distance $R_{1}$. When $d$ is larger than $R_{2}$, they will reach the equilibrium position $R_{3}$. Note, however, that the origin of position $R_{0} / 2$ in Fig. 9 is calculated numerically by a direct integration of (19). The position $R_{0}$ corresponds to the critical initial distance between spots, and below it, only one spot survives. This means they merge

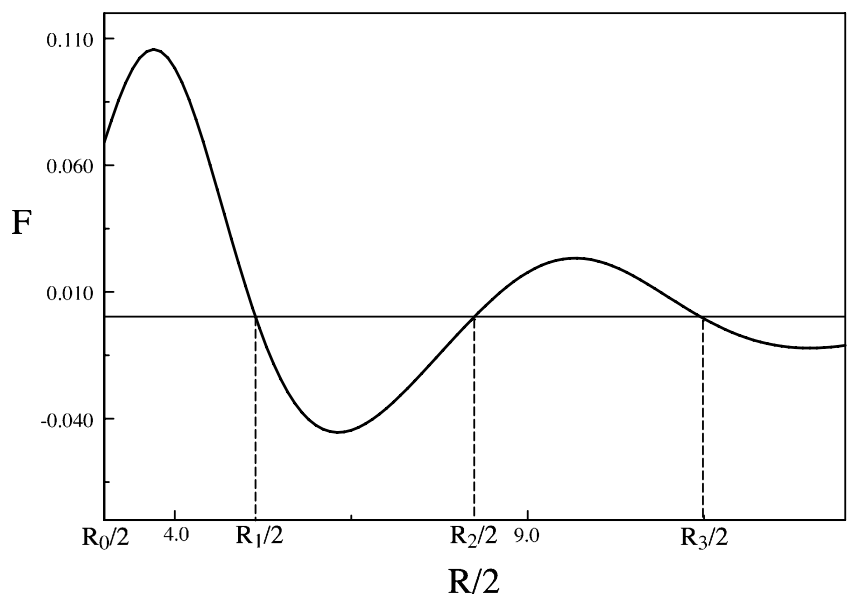

Fig. 9 Interaction force as a function of half-distance between two localized bare spots. Parameters are $\kappa=0.5, \Delta=0.1, \Gamma=2 \alpha=0.5$, and $\eta=-0.025$ 

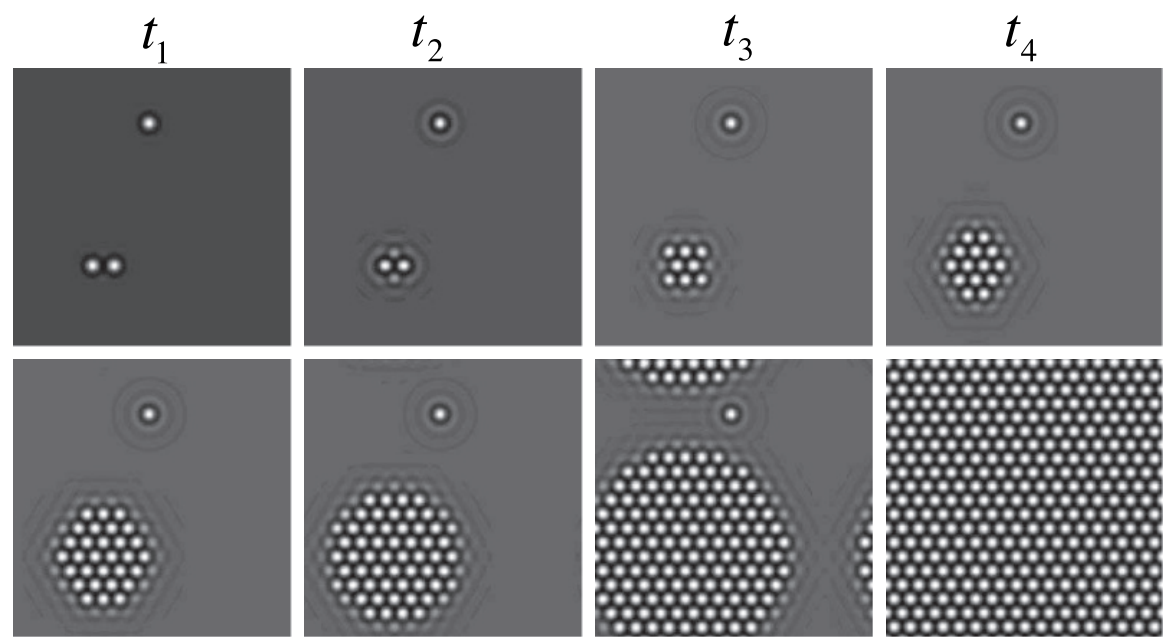

$t_{5}$

$t_{6}$

$t_{7}$

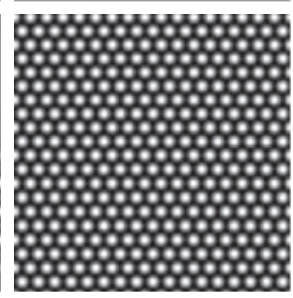

$t_{8}$

Fig. 10 Transition toward the formation of a periodic distribution of bare spots induced by the interaction between two localized bare spots. Note that the single LBS remains stable for a long time. Minima are plain white and the grid is $256 \times 256$ points. Parameters are $\kappa=0.5, \Delta=0.1$ and $\eta=-0.01\left(t_{i}=t_{i-1}+50\right.$, with $\left.t_{1}=20\right)$

if $d<R_{0}$, and only a single spot is formed. Due to the space oscillation of the tails, the interaction force alternates between attraction and repulsion, depending on the initial distance between the two bare spots.

When the branch of spot solutions coincides with the bare state (see Fig. 6), then the minimum of the spot is zero. In that case, the interaction forces between two well-separated spots will destabilize the dynamics of the system toward the formation of a periodic distribution of bare spots which will occupy the whole space available on the ground. This feature is illustrated in Fig. 10. The time evolution shows that the two LBSs become unstable under their mutual interaction, and a periodic pattern is selected in late stage evolution of the system (Fig. 10).

\section{Conclusions}

We have presented a model and nonlinear analysis which account for the clustering behaviors of arid vegetation ecosystems, the formation of localized bare soil spots (sometimes also called fairy circles) in these systems and the attractive or repulsive interactions governing their spatio-temporal evolution. The bare spots can be either spatially independent, self-organized, or randomly distributed. They form under conditions that are favorable to modulational instability, leading to the patterning. More precisely, they are generated in the regime where a periodic vegetation pattern 
and a uniform plant distribution co-exist for given, fixed values of the switching parameter $\mu$. They correspond to a spatial compromise between a homogeneous plant distribution and hexagonal patterns that consist of a uniform vegetation cover regularly punctuated with bare soil areas.

The interaction between two bare spots in 2D systems has been clarified. When the distance between them is large, bare spots do not interact. They form an isolated stationary structure. However, if they are close one to another, they will interact through their overlapping tails. The interaction will cause the bare spots to move until they reach a stable equilibrium position. We have shown that there exist several equilibrium positions. The system selects one of these positions, depending on the initial distance between the two localized spots.

The half-height width of a 2D LBS corresponds approximately to half the wavelength at the modulational instability, $\lambda_{\mathrm{m}} / 2$. The wavelength is explicitly given by a simple relation (22), where $u_{\mathrm{m}}$ is the solution of (23). The size of fairy circles ranges from 2 to $10 \mathrm{~m} \mathrm{[16].} \mathrm{The} \mathrm{spatial} \mathrm{scale} \mathrm{for} \mathrm{(19)} \mathrm{is} \mathrm{the} \mathrm{inter-plant} \mathrm{competition}$ range. This value is estimated to be of the order of $2 \mathrm{~m}$. The half-height width of a 2D LBS is of the order of three- dimensionless spatial units, and so is of order $6 \mathrm{~m}$. This value is consistent with field observations.

Acknowledgments We are grateful to N. Barbier, P. Couteron, O. Lejeune, D. Turaev, M. Wolfrum and S. Zelik for useful discussions. This research is supported, in part, by the Fonds National de la Recherche Scientifique (Belgium).

\section{References}

1. P. Greig-Smith, J. Ecol. 67, 755 (1979).

2. For a survey of this phenomenon, see e.g. Catena. 1999. The significance of Soil, Water and Landscape Processes in Banded Vegetation Patterning. 37, Special Issue, C. Valentin and J. Poesen (Editors). D. J. Tongway, C. Valentin and J. Seghieri, Banded Vegetation Patterning in Arid and Semiarid Environments, (Springer-Verlag, New York, 2001).

3. R. Lefever and O. Lejeune, Bull. Math. Biol. 59, 263 (1997).

4. O. Lejeune, P. Couteron, and R. Lefever, Acta Oecologica 20, 171 (1999).

5. R. Lefever, O. Lejeune, and P. Couteron, in: Mathematical Models for Biological Pattern Formation. IMA Volumes in Mathematics and its Applications, Frontiers in Applied Mathematics Series 121, edited by H. Othmer and P. Maini, (Springer-Verlag, New York, 2000).

6. J. von Hardenberg, E. Meron, M. Shachak, and Y. Zarmi, Phys. Rev. Lett. 87, 198101 (2001).

7. T. Okayasu and Y. Aizawa, Prog. Theor. Phys. 106, 705 (2001).

8. R. HilleRisLambers, M. Rietkerk, F. van den Bosch, H.H.T. Prins, and H. de Kroon, Ecology, 82, 50 (2001).

9. E. Gilad, J. von Hardenberg, A. Provenzale, M. Shachak, and E. Meron, Phys. Rev. Lett. 93, 098105 (2004).

10. N.M. Shnerb, P. Sarah, H. Lavee, and S. Solomon, Phys. Rev. Lett. 90, 038101 (2003).

11. M. Rietkerk, M. C. Boerlijst, F. Van Langevelde, R. HillRisLambers, J. van de Koppel, L. Kumar, H.H.T. Prins, and A.M. Roos, Am. Nat., 160, 524 (2002).

12. M. Rietkerk, S.C. Dekker, P.C. de Ruiter, and J. van de Koppel, Science 305, 1926 (2004).

13. O. Lejeune, M. Tlidi, and R. Lefever, Int. J. Quantum Chem. 98, 261 (2004). 
14. O. Lejeune, M. Tlidi, and P. Couteron, Phys. Rev. E 66, 010901(R) (2002).

15. E. Meron, E. Gilad, J. Von Hardenberg, M. Shachak, and Y. Zami, Chaos Solitons Fractals, 19, 367 (2004).

16. M.W. van Rooyen, G. K. Theron, N. van Rooyen, W.J. Jankowitz, and W.S. Matthews, J. Arid Environ. 57, 467 (2004).

17. K.A. Gorshkov and L.A. Ostrovsky, Physica D, 3, 428 (1981).

18. L.S. Aranson and K.A. Gorshkov, Physica D, 43, 435 (1990).

19. V.V. Afanasjev and N. Akhmediev, Phys. Rev. E 53, 6471 (1996).

20. N. Akhmediev, A. Ankiewicz, and J.M. Soto-Crespo, Phys. Rev. Lett. 79, 4047 (1997).

21. H.U. Bödeker, A.W. Liehr, T.D. Frank, R. Friedrich, and H.G. Purwins, New J. Phys. 6, 62 (2004).

22. A.G. Vladimirov, G.H. Khodova, and N.N. Rosanov, Phys. Rev. E 63, 056607 (2001).

23. M. Tlidi, A.G. Vladimirov, and P. Mandel, IEEE J. Quant. Elec. 39, 216 (2003).

24. A.G. Vladimirov, J.O. McSloy, D.V. Skryabin, and W. Firth, Phys. Rev. E 65, 064606 (2002).

25. R.M. Callaway and L. R. Walker, Ecology 78, 1958 (1997).

26. R. Lefever, N. Barbier, P. Couteron, and O. Lejeune, J. Ecology in preparation.

27. C.A. Klausmeier, Science 284, 1826 (1999).

28. J.A. Sherratt, J. Math. Biol. 51, 183 (2005).

29. R. Lefever, Bull. Cl. Sci. Acad. Roy. Belgique XV, 287 (2004).

30. R. Lefever, N. Barbier, P. Couteron, V. Deblauwe et O. Lejeune, Pour la Science - Dossier n 44, 68 (2004).

31. O. Lejeune and M. Tlidi, J. Veg. Sci. 10, 201 (1999); P. Couteron and O. Lejeune, J. Ecol. 89, 616 (2004).

32. S. Koga and Y. Kuramoto, Prog. Theor. Phys. 63, 106 (1980).

33. Y. Pomeau, Physica D, 23, 3 (1986).

34. S. Fauve and O. Thual, Phys. Rev. Lett. 64, 282 (1990).

35. M. Tlidi, P. Mandel, and R. Lefever, Phys. Rev. Lett. 73, 640 (1994).

36. V.B. Taranenko, K. Staliunas, and C.O. Weiss, Phys. Rev. A 56, 1582 (1997).

37. J. Boissonade, P. De Kepper, F. Gauffre, and I. Szalai, Chaos 16, 037110 (2006).

38. V.K. Vanag and I. R. Epstein, Phys. Rev. Lett. 92, 128301 (2004).

39. S. Barland, J. R. Tredicce, M. Brambilla, L.A. Lugiato, S. Balle, M. Giudici, T. Maggipinto, L. Spinelli, G. Tissoni, T. Knödl, M. Miller, and R. Jägeet, Nature 419, 699 (2002).

40. U. Bortolozzo, M.G. Clerc, C. Falcon, S. Residori, and R. Rojas, Phys. Rev. Lett. 96, 214501 (2006).

41. M. Pesch, E. Große Westhoff, T. Ackemann, and W. Lange, Phys. Rev. Lett. 95, 143906 (2005).

42. L. Yu. Glebsky and L.M. Lerman, Chaos 5, 424 (1995).

43. G.W. Hunt, G.J. Lord, and A.R. Champneys, Compt. Methods Appl. Mech. Eng. 170, 239 (1999).

44. P. Coullet, C. Riera, and C. Tresser, Phys. Rev. Lett. 84, 3069 (2000).

45. J. Burke and E. Knobloch, Phys. Rev. E 73, 056211 (2006).

46. G. Kozyreff and S.J. Chapman, Phys. Rev. Lett. 97, 044502 (2006). 